J. Indones. Math. Soc.

Special Edition (2011), pp. 89-107.

\title{
AN INTRODUCTION TO DISTANCE D MAGIC GRAPHS
}

\author{
Allen O'Neal ${ }^{1}$, Peter J. Slater ${ }^{2}$ \\ ${ }^{1}$ Mathematical Sciences Department, University of Alabama Huntsville, AL, \\ USA 35899 p65248@gdc4s.com \\ ${ }^{2}$ Mathematical Sciences Department and Computer Sciences Department, \\ University of Alabama Huntsville, AL, USA 35899 slaterp@uah.edu and \\ pslater@cs.uah.edu
}

\begin{abstract}
For a graph $G$ of order $|V(G)|=n$ and a real-valued mapping $f: V(G) \rightarrow \mathbb{R}$, if $S \subset V(G)$ then $f(S)=\sum_{w \in S} f(w)$ is called the weight of $S$ under $f$. When there exists a bijection $f: V(G) \rightarrow[n]$ such that the weight of all open neighborhoods is the same, the graph is said to be 1-vertex magic, or $\Sigma$ labeled. In this paper we generalize the notion of 1-vertex magic by defining a graph $G$ of diameter $d$ to be $D$-vertex magic when for $D \subset\{0,1, \ldots, d\}$, we have that $\sum_{u \in N_{D}(v)} f(u)$ is constant for all $v \in V(G)$. We provide several existence criteria for graphs to be $D$-vertex magic and use them to provide solutions to several open problems presented at the IWOGL 2010 Conference. In addition, we extend the notion of vertex magic graphs by providing measures describing how close a non-vertex magic graph is to being vertex magic. The general viewpoint is to consider how to assign a set $W$ of weights to the vertices so as to have an equitable distribution over the $D$-neighborhoods.
\end{abstract}

Key words: Graph Labeling, vertex magic, $\Sigma$ labeling, distance magic graphs, neighborhood sums.

2000 Mathematics Subject Classification: 05C78

Received: 09-08-2011, revised: 09-09-2011, accepted: 04-12-2012. 